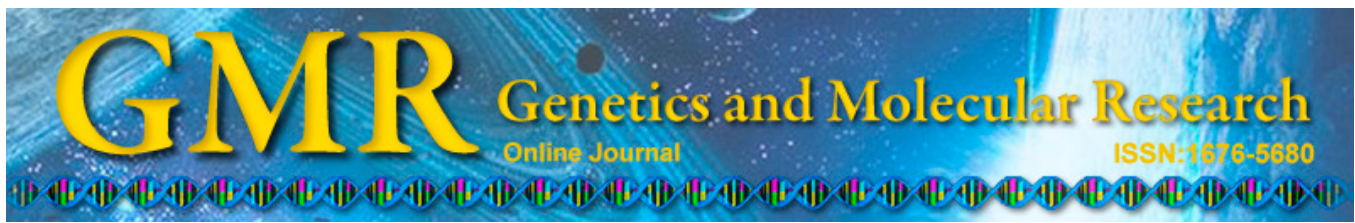

Research Note

\title{
Clinical reports and analysis of patients with clinical manifestations of migraine-like headache and unruptured aneurysm
}

\author{
M. Zhao \\ Department of Neurology, Shanghai ZhouPu Hospital, Shanghai, China \\ Corresponding author: M. Zhao \\ E-mail: zhaomei121com@126.com \\ Genet. Mol. Res. 14 (1): 1310-1317 (2015) \\ Received May 25, 2014 \\ Accepted September 11, 2014 \\ Published February 13, 2015 \\ DOI http://dx.doi.org/10.4238/2015.February.13.10
}

\begin{abstract}
A retrospective analysis of three cases of clinical manifestations of migraine-like headache, including clinical features, imaging findings, and follow-up results was done to explore the potential correlation between migraine and unruptured aneurysm. Clinical data and digital subtraction angiography (DSA) results were retrospectively analyzed. All three patients met the diagnostic criteria for migraine without aura stated in the second edition of the International Classification of Headache Disorders, established by the International Headache Society in 2004. The DSA results suggested that the aneurysms occurred in the anterior communicating artery (two cases) and in the internal carotid artery (one case); the migraine attacks disappeared after aneurysm embolization, with a follow-up time of 6 , 10 , and 16 months in the three cases, respectively. The pathogenesis of migraine is not fully understood; however, the potential correlation between migraine attack and unruptured saccular aneurysm needs attention, and the specific pathogenesis should be further investigated.
\end{abstract}

Key words: Migraine-like headache; Unruptured aneurysm; Aneurysm embolization 


\section{INTRODUCTION}

Migraine is a recurrent chronic neurological disorder. Recent epidemiological data show that the global migraine prevalence rate is about $10 \%$, and the lifetime prevalence rate is about $14 \%$. Currently, the pathogenesis of migraine is still not entirely clear; this disorder is associated with a number of factors, including a variety of physical and chemical factors, psychological factors, changes in hormone levels, and others. Here, we report the clinical data and digital subtraction angiography (DSA) results of three patients with clinically diagnosed migraine. Furthermore, we analyzed the potential correlation between migraine attack and unruptured saccular aneurysm.

\section{METHODS}

\section{Case 1}

On April 28, 2012, a 60-year-old female patient was admitted to our hospital because of repeated episodes of headache for 20 years. In 1985, she experienced a headache of no obvious source, characterized with pulsating and throbbing in the right side of her forehead. The headache was very intense and unbearable, with symptoms including nausea, phonophobia, photophobia, blurred vision, and flashing, but with no vomiting. The symptoms could only be relieved with painkillers and bed rest; otherwise, those symptoms would last for 1-2 days. The episodes of headache occurred two to three times per month with no correlation to the menstrual cycle. The patient had to carry dipyrone and Tylenol, which severely affected her daily work and life. As a result, she showed some depression. On April 17, 2012, she visited the outpatient clinic of the Department of Neurology of East Hospital. During the initial examination, cranial magnetic resonance imaging (MRI) revealed lacunar infarctions in the right semiovale area and tortuous vascular signal at the right lateral fissure cistern; cervical MRI revealed cervical degeneration in intervertebral discs $\mathrm{C} 4 / 5, \mathrm{C} 5 / 6$, and $\mathrm{C} 6 / 7$; and head magnetic resonance angiography (MRA) revealed ophthalmic artery segment protrusion in the left internal carotid artery. An aneurysm was suspected; therefore, the patient was admitted to the hospital for further DSA examination.

The patient has no history of hypertension but has had symptoms of palpitation and chest tightness since she was 9 years old. Her symptoms were worsened by exercise. In January 2009 , because of rheumatic heart disease, she underwent surgery for balloon mitral valvuloplasty. However, her mother and elder sister both have a history of migraine. During physical examination, she was conscious and could speak clearly. Her orientation, memory, and mathematical ability were normal. She showed normal appetite and sleep pattern, as well as normal urine and stool. She had no cranial nerve abnormalities; her muscle strength and tone in the limbs were normal, without atrophy or resting tremor. Superficial and deep sensations in the limbs were symmetrical. Her reflexes were normal and without pathological signs. She did not have neck stiffness, and Brudzinski's and Kernig's signs were both absent.

On May 2, 2012, the patient underwent DSA. The cerebral angiography revealed that the ophthalmic artery segment cystic protuberance on the left internal carotid artery was about $4 \mathrm{~mm}$, with wide neck aneurysm, irregular tumor size, and obvious tortuosity of the left vertebral artery. On May 7, 2012, with the microcatheter technique under admap guidance, she underwent stent-assisted coil embolization of the aneurysm under general anesthesia, by using 
an Enterprise 4.5 x 28 -mm stent (Cordis, Miami Lakes, FL, USA). The surgery was successful, and postoperative treatments such as antiplatelet aggregation were provided. On March 12, 2013, she was readmitted for examination. The angiography on March 13, 2013, showed little residual aneurysm neck at the anterior portion of the C6 segment in the left internal carotid artery. There was no contrast extravasation, the stand was in place, and blood flow was normal. The patient reported no recurrence of headache after surgical treatment.

\section{Case 2}

On September 10, 2012, a 56-year-old male patient was admitted to our hospital because of repeated episodes of headache for 20 years. The pain was intermittent with a pulsating and throbbing character, and mainly focused on the forehead. Each episode could last from several hours to 1-2 days, with symptoms including lacrimation, nasal congestion, runny nose, and convulsions; however, there were no fever and visual flashing. The headache attack had no obvious cause but was likely triggered or aggravated by fatigue and stress; rest could relieve the symptoms. During some attacks, the patient experienced severe and unbearable pain that was accompanied by nausea and vomiting. He took Saridon and headache powder during migraine attacks, and the pain was relieved after sleep. The frequency of the migraines was uncertain: sometimes once in six months, and other times several times a week.

The initial ultrasound in our clinic indicated intima-media thickness in both carotid arteries, suggesting atherosclerosis; cranial MRA revealed left anterior cerebral artery stem variation and left A2 segment aneurysm. Therefore, DSA was recommended for further diagnosis. Cranial MRI revealed pons, aged lacunar infarction in both basal ganglia and both semi-oval areas of both hemispheres, as well as ethmoid and frontal sinusitis. The patient was admitted to the hospital for further examination. It was found that he had a history of hypertension for 20 years and a 10-year history of double nephrolithiasis. On his physical examination, he was conscious and could speak clearly. His orientation, memory, and mathematical ability were normal. He had no cranial nerve abnormalities; his muscle strength and tone in the limbs were normal, without atrophy or resting tremor. Superficial and deep sensations in the limbs were symmetrical. His reflexes were normal and without pathological signs. He did not have neck stiffness, and Brudzinski's and Kernig's signs were both absent.

On September 12, 2012, under local anesthesia, the patient underwent DSA. The cerebral angiography revealed anterior communication artery fenestration, partial anterior cystic protuberance of about $3 \mathrm{~mm}$, wide neck aneurysm, irregular tumor size, and no significant intracranial arterial stenosis or expansion. A guidewire-guided $6 \mathrm{~F}$ vertebral artery catheter was used to perform arterial angiography in the internal carotid artery C2 segment. With the microcatheter technique under roadmap guidance, he underwent stent-assisted coil embolization of the aneurysm under general anesthesia by using an Enterprise 4.5 x $22-\mathrm{mm}$ stent; the angiographic aneurysm was almost occlusive and in-stent blood flow was normal. Postoperative treatments such as antiplatelet aggregation were provided. On February 19, 2013, he was readmitted for examination. The angiography showed anterior communicating artery aneurysm change after embolization, with almost no residual aneurysm neck. There was no contrast extravasation, the stent was in place, and blood flow was normal. The patient reported no recurrence of headache after surgical treatment. 


\section{Case 3}

On November 17, 2011, a 32-year-old male patient was admitted to our hospital because of repeated episodes of headaches for 5 years. The headache occurred in an episodic pattern with a pulsating and throbbing character. He experienced pain in the periorbital and temporal regions, and the headache attacks were accompanied by flu-like symptoms, including tearing eyes, nasal congestion, runny nose, and facial sweating; sometimes, the pain was accompanied by pallor and blurred vision. Each attack lasted from several hours to 1-2 days. The headache was aggravated by fatigue and stress, and rest could relieve the symptoms. During some attacks, he experienced severe and unbearable pain that was accompanied by nausea, vomiting, blurred vision, and fatigue; treatment with a strong pain analgesia was administered through the intramuscular route. The frequency of the migraines was uncertain; however, they typically occurred once or twice per month. He was examined several times at a local hospital, and was usually taking painkillers such as ibuprofen and codeine phosphate tablets.

On November 9, 2011, the patient visited the outpatient clinic of the Department of Neurology of East Hospital. An initial examination revealed normal findings for head computed tomography and MRI; however, MRA revealed a small nodule-like protuberance in the anterior communicating artery. DSA was used to confirm a diagnosis besides an aneurysm. During the physical examination, he was conscious and could speak clearly. His orientation, memory, and mathematical ability were normal. He showed normal appetite and sleep, as well as normal urine and stool. He had no cranial nerve abnormalities; his muscle strength and tone in the limbs were both normal, without atrophy and resting tremor. Superficial and deep sensations in the limbs were symmetrical. His reflexes were normal without pathological signs. He did not have neck stiffness, and Brudzinski's and Kernig's signs were both absent. During his admission, laboratory tests yielded normal blood, urine, and stool findings. Moreover, plasma glucose levels, kidney function, electrolyte levels, and serum lipid levels were all normal; human immunodeficiency virus antibody and syphilis TRUST tests were both negative. However, he was positive for hepatitis B core antibody and hepatitis B surface antibody, and negative for hepatitis C antibody. No abnormalities were seen on electroencephalography or electrocardiography. An increased number of lung markings were noted bilaterally on the chest radiograph.

The patient underwent DSA on November 18, 2011. A 2-mm cystic protuberance was identified in the superoposterior anterior communicating artery. On November 23, 2011, he underwent stent-assisted coil embolization of the aneurysm under general anesthesia by using an Enterprise $4.5 \times 14-\mathrm{mm}$ stent. Angiography was performed, and it was confirmed that the stent was patent and firmly adhered to the arterial wall. Most of the aneurysm was embolized. Postoperative treatments such as antiplatelet aggregation were provided.

On January 9, 2012, the patient was readmitted for examination. On January 11, 2012, the DSA results indicated no aneurysm recurrence after the embolization of the anterior communicating artery aneurysm, and the stent was patent and firmly adhered to the arterial wall. No recurrence of headache was reported after surgical treatment.

\section{RESULTS}

\section{Clinical features}

The disease course was chronic for all three patients, spanning 28, 20, and 5 years, 
respectively. The clinical features were recurrent headaches, from moderate to severe, that were mainly focused on a relatively fixed position. The headache had a pulsating and throbbing character accompanied by nausea and fatigue. There was no aura before the headache, and the symptoms could be relieved by taking prescription painkillers and bed rest. Each headache episode lasted from a few hours to 1-2 days; the patients' daily work and life were significantly affected by the long-term intermittent administration of painkillers. No neurological signs were observed on physical examination. According to disease history, clinical features, and ancillary examinations, all three patients met the diagnostic criteria stated in the International Classification of Headache Disorders, 2nd edition (International Headache Society, 2005), established by the Headache Classification Subcommittee of the International Headache Society in 2004, as follows: 1) presence of at least five episodes according to criteria Nos. 2 to $4 ; 2$ ) each headache attack (untreated or ineffective therapy) lasting for 4-72 h; 3) presence of at least two of the following headache characteristics: A) unilateral, B) pulsatile, C) moderate or severe pain, and D) aggravation by daily physical activity (such as walking or climbing of stairs); 4) presence of at least one of the following symptoms during a migraine attack: A) nausea or vomiting, B) photophobia and phonophobia, and C) irrelevant to other diseases.

\section{Imaging}

All three patients were observed for suspected aneurysm during cranial MRA screening in our clinic for the headache diagnosis. They were admitted to the outpatient clinic of the Department of Neurology of East Hospital and underwent DSA for further diagnostic confirmation. In case 1 , the cerebral angiography revealed that the ophthalmic artery segment cystic protuberance in the left internal carotid artery was about $4 \mathrm{~mm}$, with wide neck aneurysm, irregular tumor size, and obvious tortuosity of the left vertebral artery. In case 2, the cerebral angiography revealed anterior communication artery fenestration, partial anterior cystic protuberance of about $3 \mathrm{~mm}$, wide neck aneurysm, and irregular tumor size. In case 3, a 2-mm cystic protuberance was identified in the superoposterior anterior communicating artery with a regular tumor border. All patients underwent stent-assisted coil embolization of the aneurysm under general anesthesia. The surgeries were successful, and postoperative treatments such as antiplatelet aggregation were provided.

\section{Follow-ups}

During the outpatient follow-ups in the clinic, the migraine attacks stopped unexpectedly in all three patients after the stent-assisted coil embolization of the aneurysm. No special medication was applied unless the postoperative antiplatelet treatment was ineffective. To date, the follow-up time for the three cases is 17, 13, and 23 months, respectively. Because of the surgical correlations, the three patients were readmitted for a review of the aneurysm with DSA. The angiography of case 1 showed little residual aneurysm neck at the anterior portion of the $\mathrm{C} 6$ segment in the left internal carotid artery; on the other hand, cases 2 and 3 were both normal (Figure 1). The diagnosis was corrected as secondary headaches. 


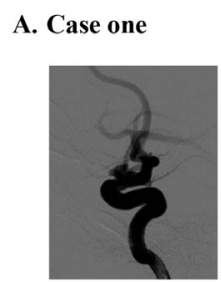

Before surgery

B. Case two

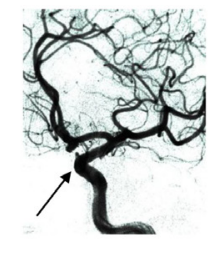

Before surgery

C. Case three

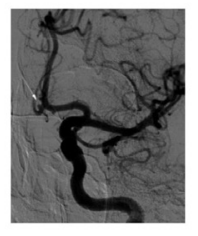

Before surgery

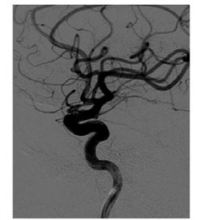

After surgery

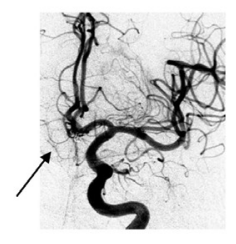

After surgery

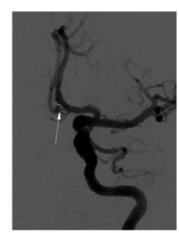

After surgery

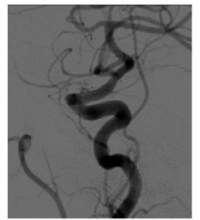

Re-examination

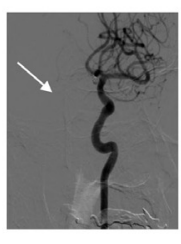

Re-cxamination

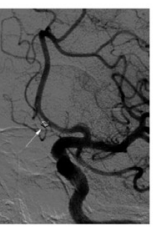

Re-examination

Figure 1. Digital subtraction angiography results of the three patients before and after surgery, and at reexamination.

\section{DISCUSSION}

According to the diagnostic criteria of the International Classification of Headache Disorders, 2nd edition (International Headache Society, 2005), headache can be attributed to cranial or cervical vascular disorders including (listed by code number) ischemic stroke (including transient ischemic attack), cerebral hemorrhage, unruptured vascular malformation (saccular aneurysms, arteriovenous malformations, dural arterovenous fistula, cavernous hemangioma), Sturge-Weber syndrome, arteritis (giant cell arteritis, primary central nervous system vasculitis, secondary central nervous system vasculitis), arterial dissection, post-endarterectomy surgery, post-carotid artery surgery, post-intracranial endovascular surgery, cerebral venous thrombosis, subcortical infarcts and leukoencephalopathy cerebral autosomal dominant arteriopathy, lactic acidosis and stroke-like episodes of mitochondrial encephalopathy, central nervous system disease, and benign pituitary apoplexy. During clinical diagnosis, it is important to pay close attention to those diseases that can cause severe headaches. Headache may be present in 17 to $23 \%$ of patients with unruptured saccular cerebral aneurysms, with nonspecific and chronic headache as the most common type, although sudden severe headache may also occur. Aneurysms $>10 \mathrm{~mm}$ can predispose the patient to thrombosis, or may cause local meningeal irritation leading to headache. Cranial nerve injury, pyramidal tract signs, 
and visual acuity or field disorders may be other symptoms of large aneurysms. Headache accompanied by oculomotor nerve palsy is the most common feature in such patients. Mydriasis can present with larger aneurysms, or with those increasing in size, which is an early sign of oculomotor nerve compression at the junction between the internal carotid artery and posterior communicating artery, at the junction between the basilar artery and superior cerebellar artery, and at the tip of the basilar artery (Bigal et al., 2004; Brandes, 2008; Wang et al., 2008).

The size of the unruptured saccular aneurysm in the three patients was 3,4 , and $2 \mathrm{~mm}$, respectively, and all patients showed no signs of cranial nerve or pyramidal tract injury. They were examined with follow-up for 17, 13, and 23 months, respectively, after the stent-assisted coil embolization of the aneurysm, and no recurrent episodes of headache were noted. At present, the pathogenesis of migraine is not entirely clear, with the trigeminal nerve vascular theory predominating (Holroyd et al., 2007; Silberstein, 2009). According to this theory, migraine is due to the dysfunction of the endogenous sensory modulation system in both the trigeminal vascular (through 5-HT1B/1D receptor modulators) and central nervous systems, plus excessive external stimulation. The distribution of dural vessels, especially intracranial vessels, is from the trigeminal nerve unmyelinated $\mathrm{C}$ fibers. These pain transmission nerve fibers have bead-like enlargement parts; when simulated electrically or chemically, aseptic inflammation will occur, including blood vessel vasodilatation, plasma protein extravasation, and mast cell degranulation. During a migraine attack, a stimulus will be generated toward the trigeminal nerve axon in the dural perivascular space, which will lead to the presence of free excitatory neuropeptides in blood vessels. As a result, local aseptic inflammation will occur. Anterograde and retrograde stimulations are transferred through the trigeminal nerve. Retrograde conduction further increases the free peptides that cause headache, and anterograde conduction increases the production of c-fos through the trigeminal nucleus, which will cause autonomic nerve system activation accompanied by nausea, vomiting and other symptoms. The intracranial sensitive structures include the dura at the bottom of the brain, and the dural artery. The aneurysm in cases 2 and 3, respectively, was in the anterior communicating artery, and in case 1, in the internal carotid artery C6 segment. Trigeminal nerve fibers are distributed in all these areas; it is still unknown whether the abnormal hemodynamic changes of aneurysm will affect the distribution of the trigeminal nerve endings, triggering a migraine attack. To our best knowledge, no similar case has been reported in the literature; therefore, further investigation is required to explore the underlying mechanisms involved (Tepper and Spears, 2009). Early diagnosis and aggressive intervention may not only reduce the risk of aneurysmal rupture but also effectively control headache. We plan to conduct long-term follow-ups for these patients, and to further investigate the relation between unruptured saccular aneurysm and migraine.

\section{Conflicts of interest}

The author declare no conflict of interest.

\section{ACKNOWLEDGMENT}

The author would like to thank the Department of Interventional Neuroradiology, Shanghai East Hospital, for providing the clinical data and digital subtraction angiography results for this study. 


\section{REFERENCES}

Bigal ME, Lipton RB and Stewart WF (2004). The epidemiology and impact of migraine. Curr. Neurol. Neurosci. Rep. 4: 98-104.

Brandes JL (2008). The migraine cycle: patient burden of migraine during and between migraine attacks. Headache 48 : 430-441.

Holroyd KA, Drew JB, Cottrell CK, Romanek KM, et al. (2007). Impaired functioning and quality of life in severe migraine: the role of catastrophizing and associated symptoms. Cephalalgia 27: 1156-1165.

International Headache Society (2005). The International Classification of Headache Disorders. 2nd ed. 1st revision. Cephalalgia 25: 460-465.

Silberstein SD (2009). Preventive migraine treatment. Neurol. Clin. 27: 429-443.

Tepper SJ and Spears RC (2009). Acute treatment of migraine. Neurol. Clin. 27: 417-427.

Wang XP, Ding HL, Geng CM and Jiang YM (2008). Migraine as a sex-conditioned inherited disorder: evidences from China and the world. Neurosci. Bull. 24: 110-116. 